\title{
Gynecologic Pathology
}

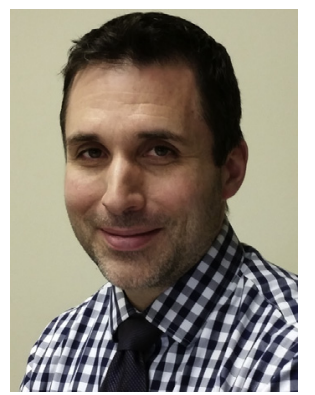

Blaise A. Clarke, MBBCh, MSc, FRCP

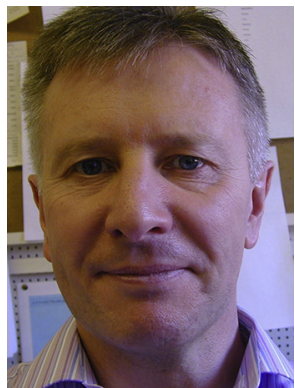

W. Glenn McCluggage, FRCPath

Editors

Molecular studies continue to validate and refine morphologic taxonomy of tumors and to explicate tumor associations. This is particularly true of hereditary predisposition in patients with cancer. With improved reproducibility in our assessment of ovarian carcinoma cell type, hereditary associations with specific histotypes have been established. Any patient with high-grade serous carcinoma of tubal/ovarian/peritoneal origin or serous tubal intraepithelial carcinoma should be referred for $B R C A 1 / 2$ germline screening given the high incidence of germline mutations in these patients (up to $25 \%$ ). Patients with synchronous or metachronous cervical embryonal rhabdomyosarcoma and ovarian Sertoli-Leydig cell tumor were reported many years ago, prompting speculation that the co-occurrence of two rare tumors may denote an underlying syndrome; it has recently become evident that both tumors may be manifestations of the DICER 1 syndrome, and their occurrence in the same patient almost certainly denotes the presence of this syndrome. Similarly, although familial small cell carcinoma of the ovary, hypercalcemic type (SCCOHT) was reported three decades ago, the underlying mechanism in both sporadic and hereditary cases of biallelic inactivation of the chromatin remodeling complex gene, SMARCA4, has only recently been elucidated; given the significant incidence of germline SMARCA4 mutations in patients with SCCOHT, genetic counseling of these patients is mandatory. We need to be aware of the growing list of tumors, which, when diagnosed in a patient, should prompt consideration of an underlying syndrome and referral to genetic counseling services.
In addition to histotype-based strategies, reflexive biomarker-driven referral strategies to detect hereditary predisposition in patients with cancer are also becoming standard of care. Reflexive testing of endometrial carcinomas (irrespective of cell type and often irrespective of patient age) with mismatch repair immunohistochemistry is being widely deployed to detect underlying Lynch syndrome. In some centers, endometrioid, clear cell, and undifferentiated ovarian carcinomas are similarly interrogated, since we now recognize a histotype-specific association between these ovarian carcinoma subtypes and Lynch syndrome.

These laboratory-driven strategies address some of the system level barriers for genetic counseling referral, such as complexity of genealogybased schemas and the poor implementation thereof, due to clinic visit time constraints and often the patient's unawareness of family medical history. Consequently, increased referral of patients to cancer-genetic consultation services is likely. There is also growing impetus for cascade testing of family members of patients found to have hereditary cancer syndromes, since screening and/or prophylactic surgery strategies in unaffected carriers have been shown to reduce morbidity and mortality in a cost-effective manner.

Increasingly, such genetic alterations transcend issues of hereditary predisposition and also convey therapeutic or predictive information, an additional catalyst for dissemination and uptake of such testing. For example, mismatch repair-deficient tumors may be targeted by immune checkpoint inhibitors such as PDL-1. PARP inhibitors are poised for clinical implementation, mandating BRCA1/2 
somatic and germline testing in patients with highgrade serous carcinoma.

In this series of articles, we highlight the critical role pathologists play in triaging patients for genetic counseling, either through the diagnosis of certain tumor types or through the reflex use of biomarkers. It behooves us as pathologists to be aware of these genotype-phenotype associations and to raise such issues in our reports, thereby directly impacting patient management. Cascade testing of family members is also yielding more prophylactic specimens from unaffected carriers, the assessment of which is critical and provides an opportunity for studying possible early or precursor lesions.

Molecular studies have not obviated histology but rather have underlined the critical importance of accurate morphologic diagnosis. The pleiotropic significance of an underlying hereditary disposition in patients with cancer is challenging how we implement genetic counseling, driving the need for clinically relevant turnaround times for these tests, and is changing the medical paradigm as we strive to balance "patient confidentiality" and "duty to warn" family members. Pathologists need to be cognizant of the diagnostic, prognostic, and therapeutic implications of these molecular alterations to determine how to deploy such testing and to ensure that, as testing algorithms become more complex and more dispersed, integration and interpretation of these findings with molecular-morphologic correlation and clinical contextualization occur.

As laboratory tests directly bespoke patient management, pathologists must be active members in the patient's circle of care, rather than relying exclusively on the vicarious pathologistpatient relationship mediated by the clinician. We need to reflect on the ethics of our technologies and consider the implication for the medical team of diagnosing or failing to diagnose hereditary predisposition in patients; this is something that has significant implications for both the patient and family members.

Blaise A. Clarke, MBBCh, MSc, FRCP

Toronto General Hospital

200 Elizabeth Street

Toronto, Ontario M5G2C4, Canada

W. Glenn McCluggage, FRCPath

Department of Pathology

Royal Victoria Hospital

Grosvenor Road

Belfast BT12BA, UK

E-mail addresses:

blaise.clarke@uhn.ca (B.A. Clarke) glenn.mccluggage@belfasttrust.hscni.net

(W.G. McCluggage) 